\title{
Offset-Free Output Feedback Predictive Control for Longitudinal Beam Dynamics in Heavy Ion Synchrotrons
}

\author{
Timm Faulwasser ${ }^{1}$, Dieter Lens ${ }^{2}$ and Christopher M. Kellett ${ }^{3}$
}

\begin{abstract}
Control of the longitudinal beam dynamics in heavy ion synchrotrons is a challenging task due primarily to the very fast time constants present in the system. Recently, the authors developed a model predictive controller for this system and demonstrated that solution of the required optimisation problem can be accomplished in about $1 \mu \mathrm{s}$ when implemented on a Field Programmable Gate Array (FPGA). This initial design made several simplifying assumptions and in this paper we extend this initial design to account for known implementation issues relevant to the SIS18 heavy ion synchrotron at the GSI Helmholtz Center. In particular, in this paper we present a design of an offset-free output feedback predictive control scheme for longitudinal beam dynamics in heavy ion synchrotrons. Furthermore, we demonstrate how to compensate for possible communication delays. The performance of this offset-free output feedback predictive controller is validated in simulation.
\end{abstract}

\section{INTRODUCTION}

Currently, more than 30,000 particle accelerators are operated around the world in both research institutions and industry for research and medical purposes. Hadron synchrotrons are high-energy accelerators for protons and ions with a closed reference orbit. Due to their fast nonlinear dynamics the operation of synchrotrons poses interesting control challenges. Here, we focus on stabilisation of the longitudinal beam dynamics in a hadron synchrotron.

Most of the existing papers dealing with beam feedback focus on hardware and implementation issues. As feedback algorithms, analog and digital filters or PI controllers are used [2, 6, 7]. Very few papers exist that focus on the feedback design. In [4], a synchrotron beam serves as an example for a robust observer based state feedback controller, but no implementation issues are discussed. In this paper, we consider the specific setup of the synchrotron SIS18 at GSI Helmholtz Center for Heavy Ion Research [5] and consider open problems that are essential for a successful implementation of Model Predictive Control (MPC) scheme.

In [3] we presented an MPC scheme for stabilisation of the longitudinal beam dynamics in the heavy ion synchrotron SIS18 at the GSI Helmholtz Center for Heavy Ion Research

$1 \mathrm{TF}$ is with the Laboratoire d'Automatique, École Polytechnique Fédérale de Lausanne, CH-1004 Lausanne, Switzerland timm. faulwasserdepfl. ch

2 DL is with the Institut für Automatisierungstechnik, Technische Universität Darmstadt, D-64283 Darmstadt, Germany dlensertr.tu-darmstadt.de

$3 \mathrm{CK}$ is with the School of Electrical Engineering and Computer Science, University of Newcastle, Callaghan NSW 2308 Australia chris.kellettenewcastle.edu.au. CK is supported by the Australian Research Council under FT1101000746. This work was also partially supported by the Alexander von Humboldt Foundation, Germany.
[5]. A Field Programmable Gate Array (FPGA) implementation of this MPC scheme, using a fast gradient method to solve the required optimisation problem, was presented in [3]. For implementation on the SIS18 synchrotron, the control action needs to be computed in under $3 \mu$ s and a timing analysis of the FPGA implementation in [3] showed that, on a high-end FPGA, the optimisation problem could be solved in approximately $1 \mu \mathrm{s}$.

The design in [3] ignored certain practical implementation issues and in this paper we address several of these issues. In particular, the current paper differs from [3] in four important aspects. First, the MPC scheme of [3] assumes full state feedback whereas here we consider the output feedback problem. Second, the MPC scheme of [3] assumes the knowledge of a set-point value whereas, in practice, the set-point is unknown a priori. Consequently, in this paper, we present an offset-free MPC design. Third, the design of [3] ignores communication and computation delays and herein we explicitly take these delays into account. Fourth, and finally, we observe that the system model in [3] contains an invariant of motion that allows us to reduce the model order for the longitudinal beam dynamics from five to four. We validate the performance of this new design by simulations.

The remainder of the paper is structured as follows: In Section II we briefly present a model of the particle bunch dynamics in synchrotrons and recall the problem of longitudinal beam feedback control. In a refinement of [3], we describe how the model for control can be reduced from five states to four states. Section III describes the proposed MPC scheme and the structures used for offset compensation and delay compensation, all making use of the refined four state model. Section IV presents results obtained from a simulation study and Section V concludes the paper.

\section{PARTICLE BEAM DYNAMICS}

\section{Longitudinal Beam Dynamics}

For constant energy, the longitudinal single-particle dynamics in hadron synchrotrons can be written as [10]

$$
\begin{aligned}
& \dot{\varphi}_{j}=-\omega_{\mathrm{syn}, 0} w_{j}, \\
& \dot{w}_{j}=\omega_{\mathrm{syn}, 0}\left(1+u_{2}\right) \sin \left(\varphi_{j}-u_{1}\right),
\end{aligned}
$$

where $j$ is the index indicating the individual particles, $\varphi$ denotes the phase or position deviation of the particles, whereas $w$ is a normalised coordinate proportional to the energy deviation. Both deviations are measured with respect to the ideal reference trajectory in the synchrotron. The frequency $\omega_{\mathrm{syn}, 0}$ is the so-called synchrotron frequency, 
which is typically in the range of a few hundred $\mathrm{Hz}$ at SIS18. The inputs $u_{1}$ and $u_{2}$ are phase and amplitude modulations.

The dynamics of a particle beam in a synchrotron are nonlinear and high-dimensional. For example, in the heavyion synchrotron SIS18 at GSI, the number of particles in the beam can exceed $10^{10}$. However, what is important for beam experiments is not the behaviour of individual particles, but the shape and properties of the overall particle ensemble. Therefore, statistical quantities may be used to model the beam dynamics. In the following, we focus on the longitudinal dynamics of the particle beam.

\section{Derivation of a Linearised Model}

A simplified model describing the longitudinal beam dynamics using moments has been derived in [11]. The model describes the dynamics of the first and second order moments of the beam. For the derivation of the model, the longitudinal single-particle dynamics (1) are used.

The state vector of the model is defined as

$$
x=\left(\begin{array}{lllll}
\rho_{\varphi} & \rho_{w} & \mu_{\varphi}-\bar{\mu}_{\varphi} & \mu_{\varphi, w} & \mu_{w}-\bar{\mu}_{w}
\end{array}\right)^{\mathrm{T}},
$$

where $\rho_{\varphi}$ and $\rho_{w}$ are the mean values of the particle ensemble, $\mu_{\varphi}$ and $\mu_{w}$ are the variances, $\mu_{\varphi, w}$ is the covariance, and $\bar{\mu}_{\varphi}$ and $\bar{\mu}_{w}$ are the set-points of $\mu_{\varphi}$ and $\mu_{w}$.

The dynamics are

$$
\dot{x}=\omega_{\text {syn }, 0}\left(\left(\begin{array}{cc}
A_{1} & 0 \\
0 & \tilde{A}_{2}
\end{array}\right) x+\left(\begin{array}{cc}
B_{1} & 0 \\
0 & B_{2}
\end{array}\right)\left(\begin{array}{l}
u_{1} \\
u_{2}
\end{array}\right)\right),
$$

with

$$
A_{1}=\left(\begin{array}{rr}
0 & -1 \\
a_{1} & 0
\end{array}\right), \tilde{A}_{2}=\left(\begin{array}{ccr}
0 & -2 & 0 \\
a_{2} & 0 & -1 \\
0 & a_{3} & 0
\end{array}\right)
$$

and

$$
B_{1}=\left(\begin{array}{r}
0 \\
-a_{1}
\end{array}\right), B_{2}=\left(\begin{array}{c}
0 \\
b_{1} \\
0
\end{array}\right)
$$

The model coefficients are functions of the set-point $\bar{\mu}_{\varphi}$ of $\mu_{\varphi}[8]:$

$$
\begin{aligned}
& a_{1}=1+\sum_{n=1}^{6} \frac{(-1)^{n}}{n ! 2^{n}} \bar{\mu}_{\varphi}^{n}, \\
& a_{2}=1+\sum_{n=1}^{6} \frac{(-1)^{n}(n+1)}{n ! 2^{n}} \bar{\mu}_{\varphi}^{n}, \\
& a_{3}=2 a_{1}, \quad b_{1}=a_{1} \bar{\mu}_{\varphi} .
\end{aligned}
$$

The model derivation involves a linearisation around the setpoint

$$
\begin{aligned}
x_{\mathrm{e}} & =\left(\begin{array}{lllll}
\rho_{\varphi} & \rho_{w} & \mu_{\varphi} & \mu_{\varphi, w} & \mu_{w}
\end{array}\right)^{\mathrm{T}} \\
& =\left(\begin{array}{lllll}
0 & 0 & \bar{\mu}_{\varphi} & 0 & \bar{\mu}_{w}
\end{array}\right)^{\mathrm{T}} \\
u_{\mathrm{e}} & =\left(\begin{array}{ll}
0 & 0
\end{array}\right)^{T},
\end{aligned}
$$

with

$$
\bar{\mu}_{w}=b_{1}=a_{1} \bar{\mu}_{\varphi}
$$

Observe that the subsystem $\left(\tilde{A}_{2}, B_{2}\right)$ contains an invariant of motion. Considering the third and fifth row of (3b)

$$
\begin{aligned}
& \dot{x}_{3}=\omega_{\mathrm{syn}, 0}\left(-2 \cdot x_{4}\right), \\
& \dot{x}_{5}=\omega_{\mathrm{syn}, 0}\left(a_{3} \cdot x_{4}\right)=-\frac{a_{3}}{2} \cdot \dot{x}_{3},
\end{aligned}
$$

shows that

$$
x_{5}(t)=-\frac{a_{3}}{2} \cdot x_{3}(t)+C
$$

holds. With the assumption that the set-point $x_{3}=x_{5}=0$ should be reachable, the constant is determined as $C=0$. A reduced version of the subsystem $\left(\tilde{A}_{2}, B_{2}\right)$ is therefore

$$
\left(\begin{array}{l}
\dot{x}_{3} \\
\dot{x}_{4}
\end{array}\right)=\omega_{\text {syn }, 0}\left(\left(\begin{array}{cr}
0 & -2 \\
a_{2}+\frac{a_{3}}{2} & 0
\end{array}\right)\left(\begin{array}{l}
x_{3} \\
x_{4}
\end{array}\right)+\left(\begin{array}{c}
0 \\
b_{1}
\end{array}\right) u_{2}\right) \text {. }
$$

In order to simplify the notation we subsequently denote the reduced state $\left(x_{1}, \ldots, x_{4}\right)^{T}$ by $x$. The reduction of (3) reads as follows

$$
\dot{x}=\omega_{\text {syn }, 0}\left(\left(\begin{array}{cc}
A_{1} & 0 \\
0 & A_{2}
\end{array}\right) x+\left(\begin{array}{cc}
B_{1} & 0 \\
0 & B_{2}
\end{array}\right)\left(\begin{array}{l}
u_{1} \\
u_{2}
\end{array}\right)\right),
$$

with

$$
A_{1}=\left(\begin{array}{rr}
0 & -1 \\
a_{1} & 0
\end{array}\right), A_{2}=\left(\begin{array}{cr}
0 & -2 \\
a_{2}+\frac{a_{3}}{2} & 0
\end{array}\right),
$$

and

$$
B_{1}=\left(\begin{array}{c}
0 \\
-a_{1}
\end{array}\right), B_{2}=\left(\begin{array}{c}
0 \\
a_{1} \bar{\mu}_{\varphi}
\end{array}\right) .
$$

The inputs are subject to the constraint

$$
\forall t: \quad u_{1}(t) \in\left[-\bar{u}_{1}, \bar{u}_{1}\right], u_{2}(t) \in\left[-\bar{u}_{2}, \bar{u}_{2}\right] .
$$

It should be noted that (8a) describes the dynamics of the whole bunch of particles (1).

\section{Beam Emittance}

A major goal of the beam feedback is the preservation of the beam quality. In order to evaluate the beam quality, we will consider the so-called rms longitudinal beam emittance. Roughly speaking, the emittance is defined as the area that is occupied by the beam in the longitudinal phase space. For arbitrary beam shapes, this area is not easy to determine. However, one simple possibility is the definition of the rms emittance [10]

$$
\epsilon_{\mathrm{rms}}=\omega_{\mathrm{syn}, 0} \sqrt{\mu_{\varphi} \mu_{w}-\mu_{\varphi, w}^{2}}
$$

where $\mu_{\varphi}$ and $\mu_{w}$ denote the variances of the particle ensemble along the axes $\varphi$ and $w$, and $\mu_{\varphi, w}$ is the covariance. In case the beam density function represents a Gaussian distribution, the emittance $\epsilon_{\mathrm{rms}}$ is the area of an ellipse containing $39.3 \%$ of all particles of the beam.

For ideal accelerators, i.e., those with only linear magnetic fields and no disturbances on the beam, the emittance is a constant of motion. However, due to various effects, the emittance increases during the acceleration cycle. The beam feedback should minimise this emittance increase. Due to the fact that the beam density function cannot be measured 
directly, the emittance can only be reconstructed off-line after the acceleration cycle and cannot be used directly for the beam feedback. Therefore, we will use the bunch moments (2) as an indirect measure for the beam quality. Our assumption is that if disturbances on the moments are quickly damped by the feedback, this will also prevent most of the emittance increase. The emittance (10) will be calculated in the simulation results of Section IV and used to justify this assumption.

\section{Formulation of the Control Problem}

The longitudinal beam feedback control problem can be summarised as follows: Stabilise the bunch moments (2), which are the outputs of the nonlinear system (1), at the steady state (5) subject to the input constraint (9).

In this paper we propose to solve the longitudinal beam feedback control problem via an offset-free MPC approach. Since the development of predictive controllers is simplified in a discrete-time setting, we discretise (8a) with zero-orderhold and a sampling time $T_{\text {samp }}=2.66 \mu$ s. This sampling time corresponds to one used for a previously developed control scheme using an FIR filter and hence allows for a fair comparison with the current state-of-the-art. The discretised version of $(8 \mathrm{a})$ reads

$$
x(k+1)=A x(k)+B u(k), \quad x(0)=x_{0} .
$$

\section{OFFSET-FREE PREDICTIVE PARTICLE BEAM CONTROL}

In order to design a realistic control scheme for the longitudinal beam feedback control problem we have to take several aspects into account:

(i) Unknown set-point: The set-point (5) is not known exactly, i.e., the constants $\bar{\mu}_{\varphi}$ and $\bar{\mu}_{\omega}=a_{1} \bar{\mu}_{\varphi}$ are unknown.

(ii) Output feedback: Full state information of system (8a) is not available. Only the moments $\rho_{\varphi}(k)$ and $\mu_{\varphi}(k)$ are available as measurements

$$
\begin{aligned}
& y_{1}(k)=\rho_{\varphi}\left(k-\delta_{m}\right)-u_{1}\left(k-\delta_{m}\right), \\
& y_{2}(k)=\mu_{\varphi}\left(k-\delta_{m}\right) .
\end{aligned}
$$

In other words, we can compute the bunch moments $\rho_{\varphi}(k)$ and $\mu_{\varphi}(k)$ directly from sensor data.

(iii) Measurement and actuation delays: Due to the need to process the measurement data, the outputs (12) are subject to a constant communication delay $\delta_{m} \in \mathbb{N}$, i.e., at time $k$ we merely know $y\left(k-\delta_{m}\right)$. Furthermore, at time $k$ the actuator only has information computed at time $k-\delta_{a}, \delta_{a} \in \mathbb{N}$. Thus, our design must account for actuation and measurement delays $\delta_{a}$ and $\delta_{m}$.

In what follows, we will describe how to account for all of these issues. First we will tackle issues (i) and (ii) via offsetfree MPC techniques (cf. [13]). Second we sketch how the measurement and computational delays can be taken into account. In order to achieve this we make the following assumptions:
(A1) The measurement delay $\delta_{m} \in \mathbb{N}$ and a possible (computational) actuation delay $\delta_{a}$ are a priori known and constant. ${ }^{1}$

(A2) The dependence of the system matrices $A_{1}, A_{2}, B_{1}, B_{2}$ on the unknown set-point $\bar{\mu}_{\varphi}$ can be neglected, i.e., in (4) we use $\bar{\mu}_{\varphi}=1$. $^{2}$

\section{Offset-free Design}

Taking (2) into account we see that (12) can be rewritten in terms of the states $x_{1}, \ldots, x_{4}$ of the reduced model

$$
\begin{aligned}
& y_{1}(k)=x_{1}\left(k-\delta_{m}\right)-u_{1}\left(k-\delta_{m}\right), \\
& y_{2}(k)=x_{3}\left(k-\delta_{m}\right)+\bar{\mu}_{\varphi} .
\end{aligned}
$$

In view of issue $(i)$ it is clear that in our MPC design we have to account for the uncertain offset $\bar{\mu}_{\varphi}$ on the output $y_{2}$.

To simplify the notation for the rest of this section we assume temporarily that the measurement delay is not present, i.e., $\delta_{m}=0$. Assuming that $\bar{\mu}_{\varphi}$ changes slowly compared to the dynamics (11) we model $\bar{\mu}_{\varphi}$ as an unknown constant disturbance $d(k) \in \mathbb{R}$ (cf. [13]). Thus we obtain the augmented system description

$$
\begin{gathered}
\left(\begin{array}{c}
x(k+1) \\
d(k+1)
\end{array}\right)=\left(\begin{array}{cc}
A & B_{d} \\
0 & 1
\end{array}\right)\left(\begin{array}{l}
x(k) \\
d(k)
\end{array}\right)+\left(\begin{array}{l}
B \\
0
\end{array}\right) u(k) \\
y(k)=\left(C, C_{d}\right)\left(\begin{array}{l}
x(k) \\
d(k)
\end{array}\right)+D u(k) .
\end{gathered}
$$

It is well-known [13] that the augmented system (14) is observable if and only if

$$
\operatorname{rank}\left(\begin{array}{cc}
A-I & B_{d} \\
C & C_{d}
\end{array}\right)=\operatorname{dim}(x)+\operatorname{dim}(d) .
$$

Assuming $B_{d}=0$ and that (A2) holds, it can be easily verified that this rank condition is satisfied. The main idea of offset-free MPC can be summarised as follows: at each time $k$ obtain the state of the augmented system (14) via an appropriate observer; solve an optimal control problem to predict the future behaviour of (14) over some horizon $N \in$ $\mathbb{N}$, and apply the first part of the optimal input trajectory.

\section{Delay Compensation via MPC}

In order to simplify the notation we write $z(k)=$ $(x(k), d(k))^{T}$ and denote the system matrix of (14a) by $\bar{A} \in \mathbb{R}^{5 \times 5}$ and $\bar{B}:=(B, 0)^{T} \in \mathbb{R}^{5 \times 2}$. Observed states are denoted by superscript $\hat{\imath}$. At each sampling instance $k$ the following optimal control problem has to be solved:

$$
\min _{\{u(i)\}} \frac{1}{2}\left\|z\left(N+\delta_{m}\right)\right\|_{P}^{2}+\frac{1}{2} \sum_{i=1}^{N+\delta_{m}-1}\|z(i)\|_{Q}^{2}+\|u(i)\|_{R}^{2}
$$

\footnotetext{
${ }^{1}$ The proposed MPC controller will eventually be implemented on an FPGA as in [3]. Thus the assumption of constant and known (computational) actuation delay is justified.

${ }^{2}$ In [11] the influence of the uncertain set-point $\bar{\mu}_{\varphi}$ on the eigenvalues of $A=\operatorname{diag}\left(A_{1}, A_{2}\right)$ is discussed. The results presented therein and our simulation results presented later justify this assumption.
} 


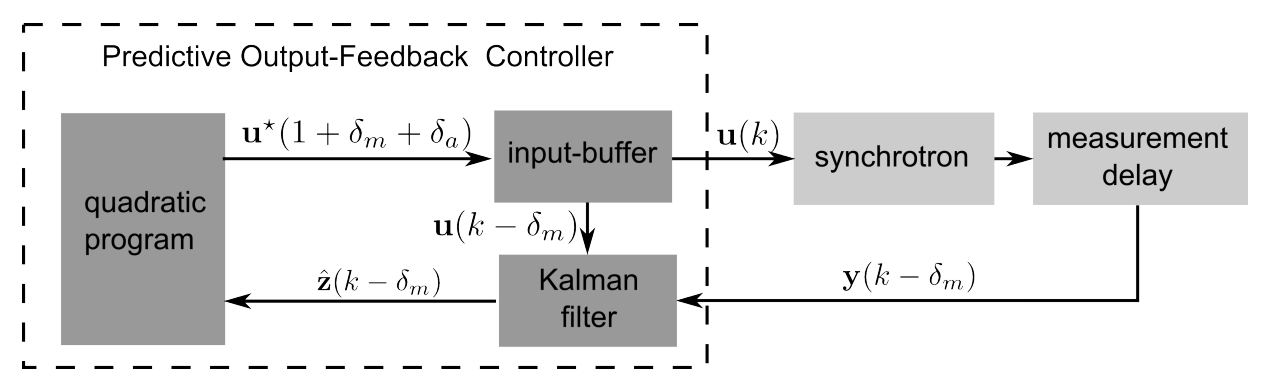

Fig. 1. Predictive output-feedback synchrotron control with measurement and actuation delays.

subject to $\forall i=0, \ldots, N+\delta_{m}-1$ :

$$
\begin{aligned}
z(i+1) & =\bar{A} z(i)+\bar{B} u(i) \\
z(0) & =\left(\hat{x}\left(k-\delta_{m}\right), \hat{d}\left(k-\delta_{m}\right)\right)^{T} \\
u(i) & \in[\underline{u}, \bar{u}]
\end{aligned}
$$

and for all $i=0, \ldots, \delta_{m}-1$ :

$$
u(i)=u^{\star}\left(1+\delta_{m}+\delta_{a} \mid \hat{z}\left(k-\delta_{m}-\delta_{a}+i\right)\right) .
$$

To simplify the notation we use here $\|z\|_{P}^{2}:=z^{T} P z$. Furthermore, $u^{\star}(l \mid \hat{z}(k))$ denotes the value of the optimal input at time $l$ based on the state estimate available at time $k$.

In principle the proposed MPC scheme is a straightforward extension to the usual QP formulation of MPC with linear dynamics, see [12, 13]. The main differences are:

(a) System (14) is subject to measurement delay, i.e., at time $k$ we only have access to the state estimate at time $k-\delta_{m}$. Hence, we set the initial conditions of the MPC prediction in (15c) as $z(0)=\hat{z}\left(k-\delta_{m}\right)$. Note that due to the measurement delay the prediction horizon is increased from $N$ to $N+\delta_{m}$.

(b) In (15e) it is enforced that the first $\delta_{m}$ elements of the decision sequence $\{u(i)\}$ are consistent with the control computed at the previous sampling instants.

While (a) follows in a straightforward manner from the presence of the measurement delay $\delta_{m}$, the equality constraints (15e) of (b) require an explanation.

Temporarily assume that the measurement and actuation delays are $\delta_{m}=1, \delta_{a}=0$ and consider the sampling instance $k$. Since $\delta_{m}=1$ the most recent state estimate at time $k$ is $\hat{z}(k-1)$. Furthermore, at time $k-1$ the input $u^{\star}(2 \mid \hat{z}(k-2))$ has been applied to the system. Hence the first element of the sequence of decision variables $\{u(i \mid \hat{z}(k-$ $1))\}$ is already fixed to $u(1)=u^{\star}(2 \mid \hat{z}(k-2))$. Generalising this observation to the case that $\delta_{m} \geq 1, \delta_{a} \geq 0$ we see that at time $k$ the first $\delta_{m}$ elements of the sequence of decision variables $\{u(i \mid \hat{z}(k))\}$ are fixed. It is this consistency requirement that is expressed in $(15 \mathrm{e})$.

Thus in the absence of actuation delay $\left(\delta_{a}=0\right)$ we apply at each sampling instance $k$ the input

$$
u(k)=u^{\star}\left(1+\delta_{m} \mid \hat{z}\left(k-\delta_{m}\right)\right) .
$$

If the actuation delay is known and constant $\delta_{a}$, we apply

$$
u(k)=u^{\star}\left(1+\delta_{m}+\delta_{a} \mid \hat{z}\left(k-\delta_{m}-\delta_{a}\right)\right) .
$$

The last equation basically means that from any optimal input sequence $\left\{u^{\star}\left(i \mid \hat{z}\left(k-\delta_{m}\right)\right)\right\}$ we do not necessarily pick the first element. Rather we pick the element with the index that corresponds to the combined measurement and actuation delay of the system. This implies that we need to store $\delta_{m}+$ $\delta_{a}$ inputs in a buffer which ensures that the correct input is applied to the system. ${ }^{3}$ The overall control algorithm is sketched in Figure 1.

Remark 1 (Implementation aspects): From an implementation point of view it may be desirable to remove the additional equality constraints (15e). This can be easily achieved by an open-loop prediction of the system state for $i=k-\delta_{m}, \ldots, k$. As discussed in [3] we use efficient fast gradient methods (FGM) [9, 14] to solve the necessary quadratic program. For FGM algorithms such simple input equality constraints can be implemented directly without any difficulty.

\section{SIMULATION RESULTS}

To assess the performance of the proposed approach we simulate the proposed MPC scheme (15). As simulated reality we consider a bunch with $\approx 2.5 \cdot 10^{5}$ particles obeying the nonlinear dynamics (1). The system is subject to measurement and actuation delay $\delta_{m}=\delta_{a}=1$. The prediction horizon is set to $N=30$. The weight matrices are chosen by trial-and-error and are set to $Q=P=$ $\operatorname{diag}(2,2,2,0,0), \quad R=\operatorname{diag}(0.9,0.5)$. We do not penalise the disturbance $d$ since this is an uncontrollable state of (14). The state $x_{4}$ is also not penalised since by comparison with a detailed model it is known that the quality of the state estimation for $x_{4}$ is not very good. The input constraints (9) are $\bar{u}_{1}=0.5, \bar{u}_{2}=0.15$. The QP (15) is solved via a Matlab implementation of the FGM with 17 iterations. ${ }^{4}$

For the sake of realistic simulation results we add uncorrelated white noise to the measurements (13). The noise level is chosen consistent with available measurement data from the SIS18 synchrotron. The state of the augmented system (14) is obtained via a standard steady state Kalman filter [1]. We consider a steady state Kalman filter since it does not require divisions or inversion of matrices which is beneficial

\footnotetext{
${ }^{3}$ Note that, even in the case of non-deterministic communication delays, the combination of buffers with MPC allows for delay compensation [15].

${ }^{4}$ This number of iterations ensures an optimality gap smaller than $10^{-6}$ and has been computed according to the accuracy bounds provided in $[9$, $14]$.
} 

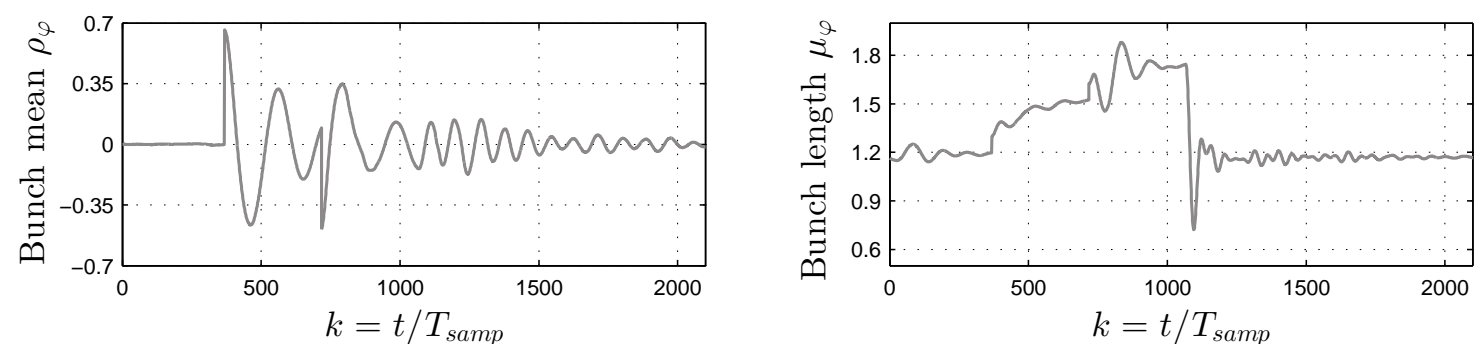

(a) Behaviour of the uncontrolled system.
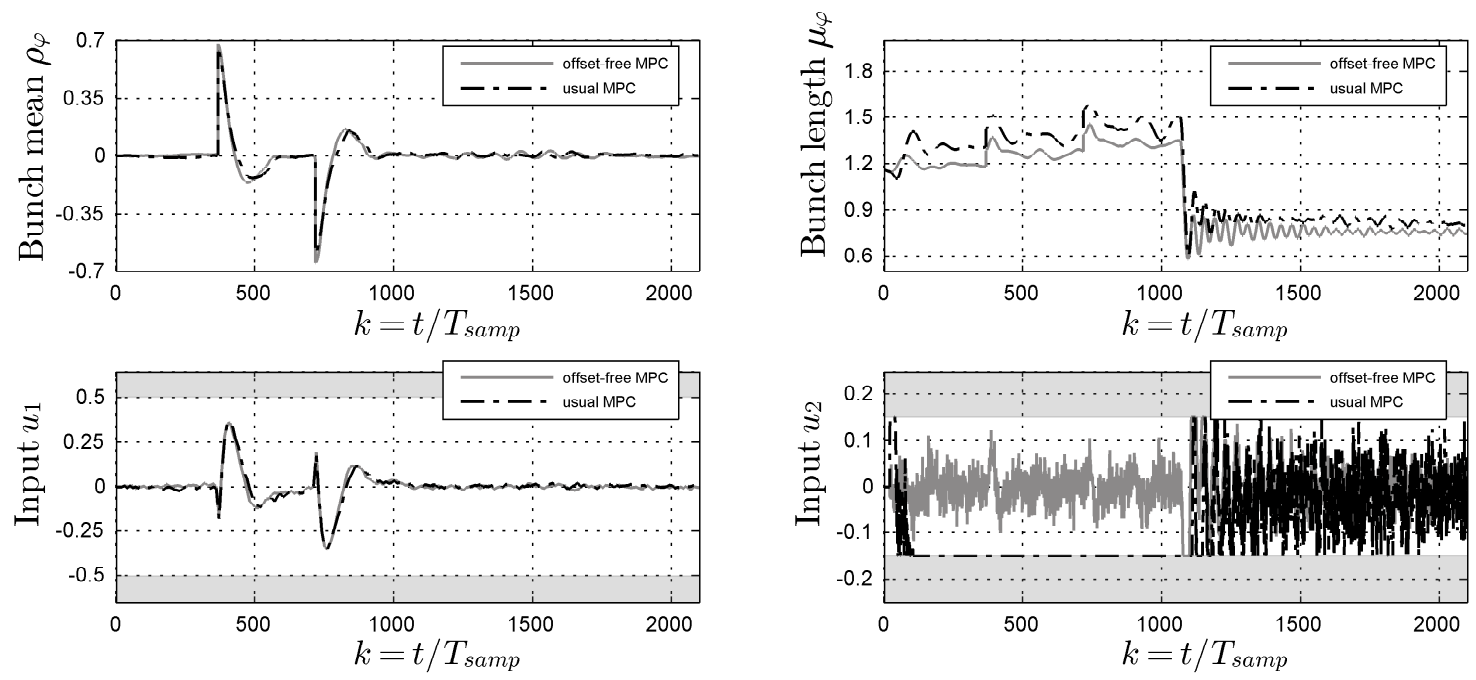

(b) Behaviour of the controlled system with a usual MPC scheme and with an offset-free MPC.

Fig. 2. Simulation results.

from an implementation perspective. Due to space limitations a detailed description of the Kalman filter is beyond the scope of this paper. The initial conditions for the observer are set to $(\hat{x}(0), \hat{d}(0))^{T}=(0,0,0,0,1)^{T}$, meaning we use $\hat{d}(0)=1$ as an initial guess for the unknown set-point. The controller is active from $k=20$ onward, where $k$ is the discretised time variable corresponding to the sampling time $T_{\text {samp }}=2.66 \mu$ s.

Figure 2 shows the behaviour of the uncontrolled system in Fig. 2(a) and the results obtained via two different MPC schemes in Fig. 2(b). In Fig. 2(b) we consider (i) the proposed offset-free MPC (15) and (ii) MPC (15) without offset compensation, i.e., $B_{d}^{T}=C_{d}=(0,0)$. In both cases state feedback is obtained from a steady state Kalman filter. At $k \in$ $\{368,718,1068\}$ we excite the nonlinear bunch dynamics (1) via jumps of the gap voltage amplitude influencing $\omega_{s y n, 0}$ or jumps of the phase angle $\varphi_{j}$ in (1). As one can see in Fig. 2(a) these disturbances lead to oscillations of the measured system outputs. These oscillations are undesirable, since they generally correspond to a deteriorated beam quality [3].

If one assumes that the unknown offset/set-point in (13) is always $\bar{\mu}_{\varphi}=1$ and applies the MPC scheme (15) without offset compensation, i.e., $B_{d}^{T}=C_{d}=(0,0)$, one obtains the results as depicted in Figure 2(b) in black dash-dot lines. One can see that although the oscillations of the bunch length

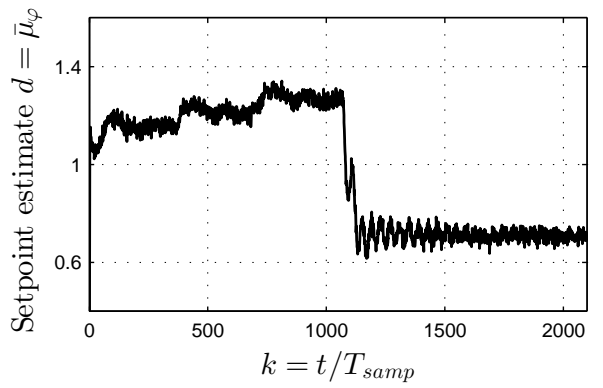

Fig. 3. Estimate of the offset/set-point $\bar{d}(k)=\bar{\mu}_{\varphi}$ obtained from the offset-free MPC scheme (15).

and the bunch mean are damped by the MPC scheme without offset compensation, the behaviour of input $u_{2}$ is undesirable since its constraints are almost always active until $k \approx 1070$. Figure 2(b) also shows the behaviour of the proposed output feedback offset-free MPC controller (15) in grey. As one can see, the amplitude of the oscillations of the bunch length is more effectively reduced compared to the MPC without offset compensation.

In Figure 3 the estimate of the offset/set-point $\hat{d}(k)=\hat{\bar{\mu}}_{\varphi}$ is depicted. Recall that we use $\hat{d}(0)=1$ as the initial guess for the unknown set-point. One can see that initially it takes 


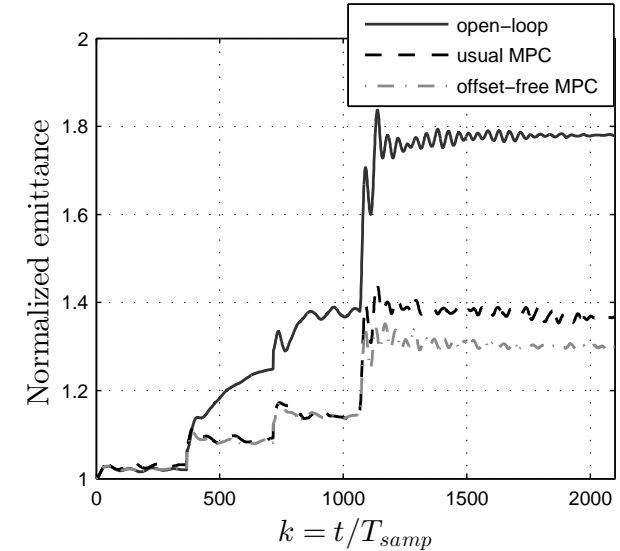

Fig. 4. Emittance for disturbed open-loop dynamics, usual MPC (without offset compensation) and the proposed offset-free MPC.

about 200 time steps until the Kalman filter converges.

Figure 4 illustrates the emittance (10) resulting from an open-loop simulation, the MPC without offset estimation, and the proposed offset-free MPC scheme. The offset-free output feedback MPC leads to low values for the emittance after $k=1068$. The MPC controller without offset estimation and the open-loop dynamics show a larger increase of the emittance. The better performance of the offset-free MPC is due the fact that the disturbance at $k=1068$ leads to a large change in the unknown set-point as shown in Figure 3. Since the offset-free MPC scheme estimates this unknown set-point it achieves better performance than the usual MPC. Finally, one can conclude that since the offsetfree design achieves lower emittance it is the preferable design for longitudinal beam feedback.

\section{CONCLUSIONS}

In this paper we presented an offset-free, output feedback predictive control approach to longitudinal beam feedback control in hadron synchrotrons. We proposed an MPC scheme that explicitly compensates for constant actuation and measurement delays. By means of simulations we have shown that the offset-free MPC approach improves the control performance. Future work will combine these results with previous investigations on the real-time feasible implementation of MPC for longitudinal beam control on FPGAs [3].

\section{BIBLIOGRAPHY}

[1] B.D.O. Anderson and J.B. Moore. Optimal Control - Linear Quadratic Methods. Information and system science series. Prentice Hall, Englewood Cliffs, London, 1990.

[2] H. Damerau, M. Paoluzzi S. Hancock, M. Migliorati, and L. Ventura. "Longitudinal coupled-bunch oscillation studies in the CERN PS". In: Proceedings of the International Particle Accelerator Conference (IPAC13), Shanghai, China. 2013.
[3] T. Faulwasser, D. Lens, and C.M. Kellett. "Predictive control for longitudinal beam dynamics in heavy ion synchrotrons". In: Control Applications (CCA), 2014 IEEE International Conference on. Antibes, France, 2014.

[4] S. Gering, J. Grieser, and A. Wahrburg. "Using LMIs to optimize robustness of observer-based statefeedback for a synchrotron". In: Int. J. Robust Nonlinear Control (2013).

[5] GSI Helmholtz Centre for Heavy Ion Research in Darmstadt, Germany. www.gsi.de.

[6] S.V. Ivanov and Q.P. Lebedev. "Analysis of the feedback-loop stability in the RF system of the U70 synchrotron". In: Instruments and Experimental Techniques 45:5 (2002), pp. 6-19.

[7] H. Klingbeil, B. Zipfel, M. Kumm, and P. Moritz. "A digital beam-phase control system for heavy-ion synchrotrons". In: IEEE Transactions on Nuclear Science 54:6 (2007), pp. 2604-2610.

[8] H. Klingbeil, D. Lens, M. Mehler, and B. Zipfel. "Modeling longitudinal oscillations of bunched beams in synchrotrons". In: arXiv preprint arXiv:1011.3957 (2010).

[9] M. Kögel and R. Findeisen. "A fast gradient method for embedded linear predictive control". In: Proceedings of the 18th IFAC World Congress, Milano, Italy. 2011, pp. 1362-1367.

[10] S.Y. Lee. Accelerator Physics. World Scientific Pub. Co., 2004.

[11] D. Lens and H. Klingbeil. "Stability of longitudinal bunch length feedback for heavy-ion synchrotrons". In: Phys. Rev. ST Accel. Beams 16:3 (2013), p. 032801.

[12] J.M. Maciejowski. Predictive control: with constraints. Pearson education, 2002.

[13] J.B. Rawlings and D.Q. Mayne. Model Predictive Control: Theory \& Design. Nob Hill Publishing, Madison, WI, 2009.

[14] S. Richter, C.N. Jones, and M. Morari. "Real-time input-constrained MPC using fast gradient methods". In: Proceedings of the 48th IEEE Conference on Decision and Control and the 28th Chinese Control Conference, CDC/CCC, Shanghai, China. 2009, pp. 7387-7393.

[15] P. Varutti, B. Kern, T. Faulwasser, and R. Findeisen. "Event-based model predictive control for networked control systems". In: Proc. 48th IEEE Conference on Decision and Control held jointly with the 28th Chinese Control Conference CDC/CCC. Shanghai, China, 2009, pp. 567-572. 